\title{
The Effect of Static Ear Canal Pressure on Human Spontaneous Otoacoustic Emissions: Spectral Width as a Measure of the Intra-cochlear Oscillation Amplitude
}

\author{
Pim van Dijk ${ }^{1,2}$, Bert MaAT ${ }^{1,2}$, And Emile de Kleine ${ }^{1,2}$ \\ ${ }^{1}$ Department of Otorhinolaryngology/Head E Neck Surgery, University Medical Center Groningen, P.O. Box 30001, 9700 RB \\ Groningen, The Netherlands \\ ${ }^{2}$ School of Behavioral and Cognitive Neurosciences, Faculty of Medicine, University of Groningen, Groningen, The Netherlands
}

Received: 21 December 2009; Accepted: 30 September 2010; Online publication: 9 November 2010

\begin{abstract}
Spontaneous otoacoustic emissions can be detected as peaks in the Fourier spectrum of a microphone signal recorded from the ear canal. The height, center frequency, and spectral width of SOAE peaks changed when a static pressure was applied to the ear canal. Most commonly, with either increasing or decreasing static pressure, the frequency increased, the amplitude decreased, and the width increased. These changes are believed to result from changes in the middle ear properties. Specifically, reduced middle ear transmission is assumed to attenuate the amplitude of emissions. We reconsidered this explanation by investigating the relation between peak height and width. We showed that the spectral width of SOAE peaks is approximately

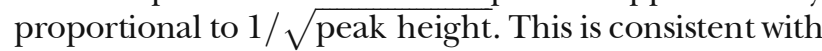
a (Rayleigh) oscillator model in which broadening of the SOAE peak is caused by broadband intra-cochlear noise, which is assumed to be independent of static ear canal pressure. The relation between emission peak height and width implicates that the intra-cochlear oscillation amplitude attentuates relative to the intracochlear noise level when a static ear canal pressure is applied. Apparently, ear canal static pressure directly affects the active mechanics in the inner ear.
\end{abstract}

Correspondence to: Pim van Dijk · Department of Otorhinolaryngology/Head \& Neck Surgery · University Medical Center Groningen · P.O. Box 30001, 9700 RB Groningen, The Netherlands. Telephone: +31-50-3612550; fax: +31-50-3611698; email: p.van.dijk@med.umcg.nl
Keywords: human, spontaneous otoacoustic emissions, middle ear, Rayleigh oscillator

\section{INTRODUCTION}

The amplitude and frequency of spontaneous otoacoustic emissions are affected by static pressure in the ear canal (Schloth and Zwicker 1983; Hauser et al. 1993; Wada et al. 1995). Both an increase and a decrease of the static pressure typically result in an increase of the emission frequency and a decrease of the emission amplitude. These effects are believed to result from changes of the middle ear impedance. Stiffening of the tympanic membrane due to static ear canal pressure may result in a change (probably stiffening) of the ligaments that connect the stapes to the round window. This changes the impedance load on emission generators in the cochlea.

In addition to the description of SOAE behavior in terms of amplitude and frequency, a spectral SOAE peak in a recording spectrum can also be characterized by its width. In humans, the width of emission peaks ranges from 0.15 to $40 \mathrm{~Hz}$ (Bialek and Wit 1984; Van Dijk and Wit 1990a; Talmadge et al. 1993). The width reflects random frequency fluctuations that presumably result from interactions between the emission generators and intra-cochlear noise. In a generic model of self-sustained oscillation, the Rayleigh oscillator, the width of the oscillation peak is proportional to the signal-to-noise ratio (Stratonovich 1963). In other words, the width of an oscillation peak can be taken as a measure of the signal-to-noise ratio. Hence, we will 
interpret the width of a spontaneous otoacoustic emission spectral peak as a measure of the intracochlear signal-to-noise ratio.

We explored the properties of peaks in SOAE spectra while changing static ear canal pressure. This gave a comprehensive description of the effect of pressure on SOAE amplitudes and frequencies that was consistent with earlier reports (Schloth and Zwicker 1983; Hauser et al. 1993). In addition, we show that emission amplitudes were inversely correlated with corresponding spectral widths. This result is interpreted in the context of the Rayleigh oscillator model. If we assume the intra-cochlear noise to be independent of the static ear canal pressure, our result implies that the intra-cochlear emission source signal must have changed. Apparently, application of static pressure directly influences the intra-cochlear active mechanics.

\section{THEORY}

The frequency spectrum of a spontaneous emission recording may contain several narrow peaks, each corresponding to a nearly sinusoidal signal emitted from the ear (Bialek and Wit 1984; Van Dijk and Wit 1990a; Talmadge et al. 1993). The behavior of these peaks has been described by a Van der Pol oscillator model. Such a model captures many characteristics of individual peaks in the emission spectrum. For example, the dynamics of amplitude relaxation is well described by a Van der Pol oscillator (Murphy et al. 1995a), as is the coupled relaxation of multiple SOAEs (Murphy et al. 1995b). Also, phase locking of an SOAE peak to a nearby external stimulus can be modeled by a Van der Pol oscillator (Van Dijk and Wit 1990b).

The amplitude and frequency of SOAEs show small fluctuations relative to their average value (Bialek and Wit 1984; Van Dijk and Wit 1990a). These fluctuations can be modeled by considering an oscillator that is exposed to a noise source:

$$
m \ddot{x}-\left[R_{1}-R_{2} \dot{x}^{2}\right] \dot{x}+k x=\eta(t)
$$

where $x(t)$ is the oscillation amplitude. The oscillator described by this equation is known as the Rayleigh oscillator. In the conditions for which the derivations below are given, the behavior of the Rayleigh and more commonly used Van der Pol oscillators is essentially identical. Although the Van der Pol oscillator has frequently been used to describe SOAEs, we chose to follow the mathematical derivations given by Stratonovich (1963), who focused on the Rayleigh oscillator.

In the force Eq. 1 a mass $m$ is assumed to be attached to a spring with constant $k$. The term $-\left[R_{1}-R_{2} \dot{x}^{2}\right]$ is the nonlinear damping typical of the
Rayleigh oscillator. ${ }^{1}$ The negative damping term $-R_{1} \dot{x}$ serves as the energy source of the oscillator. The positive term $R_{2} \dot{x}^{3}$ drains energy from the oscillator. Its small value at small amplitude $x$ and its rapid growth for larger deviations, ensures that the oscillation amplitude converges to a stable value.

The term $\eta(t)$ is the inherent noise to which the oscillator is exposed. It represents influences on the oscillator that destabilize the amplitude and frequency. In the case of SOAEs, it may represent thermal noise in the cochlea to which an emission generator is exposed (Van Dijk and Wit 1990a). However, it may also represent noise that is related to for example blood flow, to account for periodic fluctuations observed in SOAE signals (Bell 1992; Long and Talmadge 1997). Here, we only consider broadband Gaussian noise as the disturbing force $\eta(t)$ in Eq. 1, since the resulting spectral properties best describe the observed emission spectra.

For further computations, it is convenient to divide the force Eq. 1 by $m$, which results in a normalized equation of motion

$$
\ddot{x}+\omega_{0}^{2} x=\varepsilon \omega_{0}\left[1-\frac{4 \dot{x}^{2}}{3 \omega_{0}^{2} A_{0}^{2}}\right] \dot{x}+\omega_{0}^{2} \xi(t) .
$$

In this equation, three new variables $\omega_{0}, \varepsilon, A_{0}$ and a new noise term $\xi(t)$ were introduced:

$$
\begin{gathered}
\omega_{0}=\sqrt{k / m} \\
\varepsilon=\frac{R_{1}}{\sqrt{k m}} \\
A_{0}=\frac{1}{\omega_{0}} \sqrt{\frac{4 R_{1}}{3 R_{2}}} \\
\xi(t)=\eta(t) / k
\end{gathered}
$$

In general, the equation of motion of the Rayleigh oscillator does not have an exact solution, due to the nonlinear damping term that includes $\dot{x}^{2}$. However, several approximations can be made, which are relevant for the description of spontaneous otoacoustic emissions. It is convenient to write the solution of the normalized Eq. 2 as

$$
x(t)=A(t) \cos \left[\omega_{0} t+\phi(t)\right]
$$

\footnotetext{
1 The damping equals $-\left[R_{1}-R_{2} x^{2}\right]$ in the case of a Van der Pol oscillator.
} 
where the amplitude $A(t)$ and the phase $\phi(t)$ are defined in the Appendix. In general, the amplitude and the phase are fluctuating functions. For the description of spontaneous otoacoustic emissions, we may consider conditions for which the Rayleigh oscillator exhibits a nearly sinusoidal oscillation. This is the case, if $\varepsilon \ll 1$, which implies that the damping term is small, relative to the other terms in the equation of motion. Since the oscillation is nearly sinusoidal, the amplitude $A(t)$ and $\phi(t)$ must be slowly varying functions (see Stratonovich 1963). In absence of the noise term, the oscillator exhibits a stable "spontaneous" oscillation (see the Appendix):

$$
x(t) \approx A_{0} \cos \left[\omega_{0} t+\phi_{0}\right],
$$

where $\phi_{0}$ is an arbitrary constant. Note that although $\varepsilon$ is small, the amplitude $A_{0}$ is not necessarily small. As follows from the definition of $A_{0}$ (Eq. 5), the oscillation amplitude depends on the ratio between $R_{1}$ and $R_{2}$ : the oscillation amplitude increases when the negative term of the damping increases, and the amplitude decreases when the positive (nonlinear) term of the damping increases.

The oscillator no longer maintains a stable oscillation amplitude and phase, when noise is present $(\xi(t) \neq 0)$. If the disturbing noise is wideband Gaussian noise, the most conspicuous effect of the noise is that the phase $\phi$ $(t)$ diffuses freely. As a consequence, the peak in the oscillator spectrum broadens and takes the shape of a Lorentzian curve (see Appendix, Eq. 40):

$$
S_{x}(\omega) \approx \frac{A_{0}^{2}}{4} \frac{D}{\left(\omega-\omega_{0}\right)^{2}+\frac{1}{4} D^{2}},
$$

where $D$ is referred to as the phase diffusion constant. It is a measure of the amount of phase diffusion and it equals the width $\Delta \omega$ of the spectral peak. The phase diffusion constant is given by (see Appendix)

$$
\Delta \omega=D=\frac{\omega_{0}^{2} S_{\xi}\left(\omega_{0}\right)}{2 A_{0}^{2}}, \quad \text { "Width" }
$$

where $S_{\xi}$ is the spectral density of the disturbing noise. Thus, with an increasing noise level, the width of the spectrum increases. Also, with decreasing oscillation amplitude $A_{0}$ the width increases.

By combining Eqs. 9 and 10, we can derive a simple relation between the parameters that describe the spectrum of the oscillator. Note that the peak height $S_{x}\left(\omega_{0}\right)$ of the oscillator spectrum follows straightforwardly from the spectrum Eq. 9:

$$
S_{x}\left(\omega_{0}\right)=\frac{A_{0}^{2}}{D}, \quad \text { "Height }{ }^{\prime \prime}
$$

and consequently

$$
\frac{\Delta \omega}{\omega_{0}}=\sqrt{\frac{S_{\xi}\left(\omega_{0}\right)}{2 S_{x}\left(\omega_{0}\right)}} .
$$

Thus, the larger the spectral peak height $S_{x}\left(\omega_{0}\right)$, the smaller $\Delta \omega$, e.g. the narrower the peak. Note that the equation contains three quantities that can be measured in the case of an SOAE peak in an emission spectrum: the center frequency $\omega_{0}$ and the peak width $\Delta \omega$ follow directly from the spectrum. The intracochlear peak spectral density $S_{x}\left(\omega_{0}\right)$ is proportional to the peak height in an emissions spectrum recorded in the ear canal.

Equation 12 will be used to relate the width of SOAE peaks to the peak height. By applying static pressure to the external ear canal, the amplitude of an SOAE peak may change (Schloth and Zwicker 1983; Hauser et al. 1993; Wada et al. 1995). If the SOAE amplitude changes as a result of a change in the intra-cochlear source signal, this will correspond to a change of the intra-cochlear emission spectral density $S_{x}(\omega)$. Under the assumption that the intracochlear noise $\left(S_{\xi}(\omega)\right)$ is independent of the static ear canal pressure, the relation between emission peak width and peak height is predicted to be

$$
\Delta \omega \propto \frac{1}{\sqrt{S_{x}\left(\omega_{0}\right)}}, \quad \text { "Width } \propto \frac{1}{\sqrt{\text { Height }}} "
$$

where the small shifts of the SOAE frequency $\omega_{0}$ (typically $\sim 1 \%$; see Schloth and Zwicker 1983; Hauser et al. 1993; Wada et al. 1995) have been omitted for simplicity. In contrast, if the SOAE amplitude changes as a result of a changed middle ear transmission, while the amplitude of the intra-cochlear emission source signal remains unchanged, the relation becomes

$$
\Delta \omega=\cdot C \text { "Width }=\text { Constant }{ }^{\prime \prime}
$$

By evaluating the relation between peak height and width, we identified whether the behavior of SOAEs is consistent with either Eq. 13 or Eq. 14. If Eq. 13 applies, the SOAE amplitude changes are consistent with a change of the intra-cochlear source signal of the otoacoustic emission. This shows that static ear canal pressure, and hence that status of the middle ear, directly influences the active mechanics of the inner ear. In contrast, if the SOAE peak width is constant while the peak height changes (Eq. 14), the SOAE amplitude changes are likely to be due to a change of the middle ear transmission ratio. 


\section{MATERIAL AND METHODS}

Spontaneous otoacoustic emissions (SOAEs) were recorded in eight female human subjects with age ranging from 23 to 49 years (median 26.5 years). All subjects were normal hearing and showed normal tympanograms. The emissions were recorded using an ER10C high-sensitivity microphone (Etymotic Research Inc., Elk Grove Village, IL, USA). The microphone was built into a custom probe that could be tightly sealed to the ear canal using a flexible plastic tip. In addition, the probe was connected to the air pump of a TympStar clinical tympanometer (Grason-Stadler Inc., Milford, NH, USA) to control the static ear canal pressure. The pressure could be controlled with a resolution of $5 \mathrm{daPa}$.

All emission signals were amplified $40 \mathrm{~dB}$ by the ER10C pre-amplifier. Signals were digitized for offline analysis, using the built-in A/D converter of an Apple G5 computer, after additional amplification by a SR 560 low-noise amplifier (Stanford Research Systems, Sunnyvale, CA, USA). The amplification of this amplifier was set as high as possible (typically $2-5 \times$ ), without overloading its own output circuit and the computer's A/D converter. The filter of the amplifier was set to bandpass from $300 \mathrm{~Hz}$ to $10 \mathrm{kHz}$ with $6 \mathrm{~dB}$ / oct rolloffs. The A/D converter of the computer digitized the recording signal at a $48-\mathrm{kHz}$ sampling rate with a 16 -bit resolution.

SOAE recordings were made for consecutive settings of the static ear canal pressure. For each pressure setting (controlled by the tympanometer), a 1-min recording was stored on the computer disk. The initial pressure was $0 \mathrm{daPa}$ (atmospheric pressure). In four subjects, the pressure was regulated upward to $200 \mathrm{daPa}$, then down to $-200 \mathrm{daPa}$ and back to $0 \mathrm{daPa}$, all in steps of $50 \mathrm{daPa}$ for the subsequent recordings. In four other subjects, the order of pressures was reversed, with the negative pressures taken first, followed by the positive pressures. Care was taken for an appropriate seal of the microphone probe, such that the air pump did not need to be active during the 1-min recordings. A total of 17 recordings was made for each subject, each corresponding to a particular ear canal pressure.

Average frequency spectra were computed offline, using $50 \%$ overlapping time windows (Hanning). A window was rejected if the signal crossed a pre-set threshold. The squared absolute value of the FFT of each window was computed, and averaged across nonrejected windows. This gave an estimate of the power spectrum of the recorded signal. Average spectra were computed for a range of window lengths, from $n=$ 16,384 to 524,288 data points. This corresponds to a frequency resolution from 2.69 to $0.085 \mathrm{~Hz}$.
The FFT spectra are interpreted as a measure of spectral density, that has $\mathrm{Pa}^{2} / \mathrm{Hz}$ as its unit. Spectra were converted to a $\mathrm{dB}$ scale. That is, the logarithm of the power spectrum was taken and is multiplied by 10 . The $\mathrm{dB}$ scale was normalized such that $0 \mathrm{~dB}$ corresponds to the power spectrum level of a signal at $0 \mathrm{~dB}$ re $20 \mu \mathrm{Pa}$, analyzed in a $1-\mathrm{Hz}$ bandwidth. Thus, the unit on the vertical axis is $\mathrm{dB}$ re $400 \times 10^{-12} \mathrm{~Pa}^{2} / \mathrm{Hz}$. In the plots in this paper, the more commonly used $20 \times 10^{-6} \mathrm{~Pa} / \sqrt{\mathrm{Hz}}$ has been noted as the reference.

The average spectra consisted of a noise floor with SOAE peaks at particular frequencies. SOAE peaks in the spectrum were least-squares fitted to a Lorentzian curve that was also converted to the $\mathrm{dB}$ scale:

$$
\begin{aligned}
& I\left(L_{0}, f_{0}, \Delta f, C ; f\right)= \\
& 10 \times \log _{10}\left[\frac{L_{0}}{1+4\left(\frac{f-f_{0}}{\Delta f}\right)^{2}}+C\right],
\end{aligned}
$$

in order to determine the peak height $L_{0}$, center frequency $f_{0}$, and width $\Delta f$. The constant term $C$ reflects the noise floor. SOAE peaks within each subject differed with respect to their spectral width. For each peak, the spectrum with a frequency resolution was chosen, for which at least ten spectral points were contained in the main portion of the peak. By making curve fits for the spectra that corresponded to a range of ear canal pressures, the behavior of each SOAE peak was determined.

In order to provide a more comprehensive description of the effect of static ear canal pressure on the amplitude and frequency of SOAEs, we applied principal component analysis (PCA). As an example, consider the relation between SOAE frequency and static pressure. The SOAE peaks for which data were available for all 17 pressure settings, and for which the frequency jump was less than $10 \mathrm{~Hz}$ between successive recordings, were included in the PCA analysis. As will be described in the Results section, 25 peaks met this requirement. Consequently, a total of $25 \times 17$ frequency estimates entered the PCA. The frequency values were denoted by $f_{i, j}$, where $i=1, \cdots, 25$ identifies each of the peaks and $j=1, \cdots, 17$ corresponds to the static pressure. The first principal component is the vector of 17 frequency values $\mathcal{F}_{1, j}$ that is determined by minimizing the least-squares sum

$$
S=\sum_{i=1}^{25} \sum_{j=1}^{17}\left(f_{i, j}-b_{1, i} \mathcal{F}_{1, j}\right)^{2}
$$

In the minimization procedure, all the terms $b_{1, i}$ and $\mathcal{F}_{1, j}$ are varied. Note that in the result of this 
procedure, the first principal component $\mathcal{F}_{1, j}$ is the same for all 25 peaks, and each peak has its individual factor $b_{1, i}$. In this sense, the first principal component shows the behavior that is common across the 25 emission peaks, and describes most of the variation induced by static pressure changes. Note also, that the 17 values $\mathcal{F}_{1, j}$ each correspond to an ear canal pressure in the range -200 to $+200 \mathrm{daPa}$. Thus, the component can be shown in a graph that has pressure on the horizontal axis, and frequency (that is $\mathcal{F}_{1, j}$ ) on the vertical axis.

After determining the first principal component, the second component can be obtained by repeating the procedure on the residuals. Iterating the procedure may eventually provide 17 principal components. However, in practice, PCA is not employed by an iterative minimization procedure. Rather, the principal components are computed by singular value decomposition of the $25 \times 17$ data matrix.

Once the principal components are determined, the extent to which a particular component represents the behavior of an individual SOAE peak can be assessed by a factor analysis. We limited our factor analysis to the first and second principal components. The relative contribution of the first and second principal components to the behavior of individual SOAE peaks was determined by minimizing

$$
S_{i}\left(b_{1}, b_{2}\right)=\sum_{j=1}^{17}\left(f_{i, j}-b_{1} \mathcal{F}_{1, \mathrm{j}}-b_{2} \mathcal{F}_{2, \mathrm{j}}\right)^{2},
$$

where $f_{i, j}$ represents the SOAE frequency for the $i$ th emission peak at the $j$ th pressure setting $(i=1, \cdots, 17) . \mathcal{F}_{1}$ and $\mathcal{F}_{2}$ are the normalized principal components. Unlike "standard" principal component analysis, we here normalized each principal component such that its value at $-200 \mathrm{daPa}$ equalled +1 . Hence, the factors $b_{1}$ and $b_{2}$ are in Hertz and reflect frequency shifts at $-200 \mathrm{daPa}$.

For each SOAE peak frequency that could be tracked over all 17 pressure values, the factors $b_{1}$ and $b_{2}$ were determined. Analogously, the principal components $\mathcal{A}$ were determined from the amplitude data. The 17 amplitude values of the 25 SOAE peaks were combined in a $25 \times 17$ matrix $A_{i, j}$. Singular value decomposition provided 17 principle components. The relative contribution of the first two amplitude components was determined by minimization of the sum

$$
S_{i}\left(a_{1}, a_{2}\right)=\sum_{j=1}^{17}\left(A_{i, j}-a_{1} \mathcal{A}_{1, \mathrm{j}}-a_{2} \mathcal{A}_{2, \mathrm{j}}\right)^{2},
$$

where $a_{1}$ and $a_{2}$ are the factor strengths for amplitude, and $\mathcal{A}_{1}$ and $\mathcal{A}_{2}$ are the first and second principal components, respectively.
Together, this analysis allowed for a systematic description of SOAE behavior. The frequency factors $b_{1}$ and $b_{2}$ and the amplitude factors $a_{1}$ and $a_{2}$ were correlated with the frequency and level of SOAEs at the initial pressure $(0 \mathrm{daPa})$. When a Pearson correlation with frequency was computed, we always used $\log$ (frequency).

\section{RESULTS}

For each subject, one ear was investigated. The number of SOAE peaks per ear ranged from 2 to 15 , with an average of 5.1 peaks. A total of 41 SOAE peaks were included in the analysis. The peak frequencies ranged from 923 to 5,883 $\mathrm{Hz}$. The peak levels ranged from -12.1 to $18.2 \mathrm{~dB}$ SPL, with an average of $-2.3 \mathrm{~dB}$ SPL. Figure 1 shows two example spectra of SOAEs. For the subject of Figure 1A, three peaks were analyzed. For the subject of panel (B) 15 peaks were analyzed. The range of SOAE frequencies and levels across subjects is demonstrated in panel $(\mathrm{C})$, along with the noise level of the recording setup.

For all peaks that were analyzed, the peak center frequency, height and width were determined by fitting a Lorentzian curve to the spectrum. Nearly all peaks fitted well with a single Lorentz curve. An example is shown in Figure 2. In some exceptional cases, where SOAE peaks were very close together, the sum of two Lorentz curves was used as a model function.

In one case, a Lorentz curve did not represent well the shape of the SOAE peak at some of the positive static pressures. This was the case for the strongest peak we detected. At $p=-50 \mathrm{daPa}$ (where the SOAE did resemble a Lorentz peak) the frequency was $1839.9 \mathrm{~Hz}$, the width $0.1 \mathrm{~Hz}$, and the level $18.8 \mathrm{~dB}$ SPL. This peak will not be further considered in this paper. Hence, below, we will present results only for the remaining 40 SOAE peaks.

Figures 3 and 4 show an example of the most common behavior of SOAE peaks. Both with increasing and decreasing ear canal pressure, the frequency of this peak increased and its amplitude decreased. The dependence of both frequency (Fig. 4A) and peak height (Fig. 4B) is roughly symmetric about a negative static pressure, -50 daPa in this case. Panel (C) shows that the peak width also depends on static ear canal pressure: along with decreasing SOAE peak height, the width tended to increase. This relation is illustrated in panel (D): the SOAE peak height is approximaly

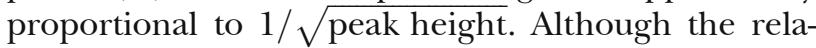
tion between peak height and width closely follows this relation for some of the pressure values, also irregular deviations from this behavior were observed, as can be seen by the deviation between some of the data points in Figure 4D and the dashed line. 

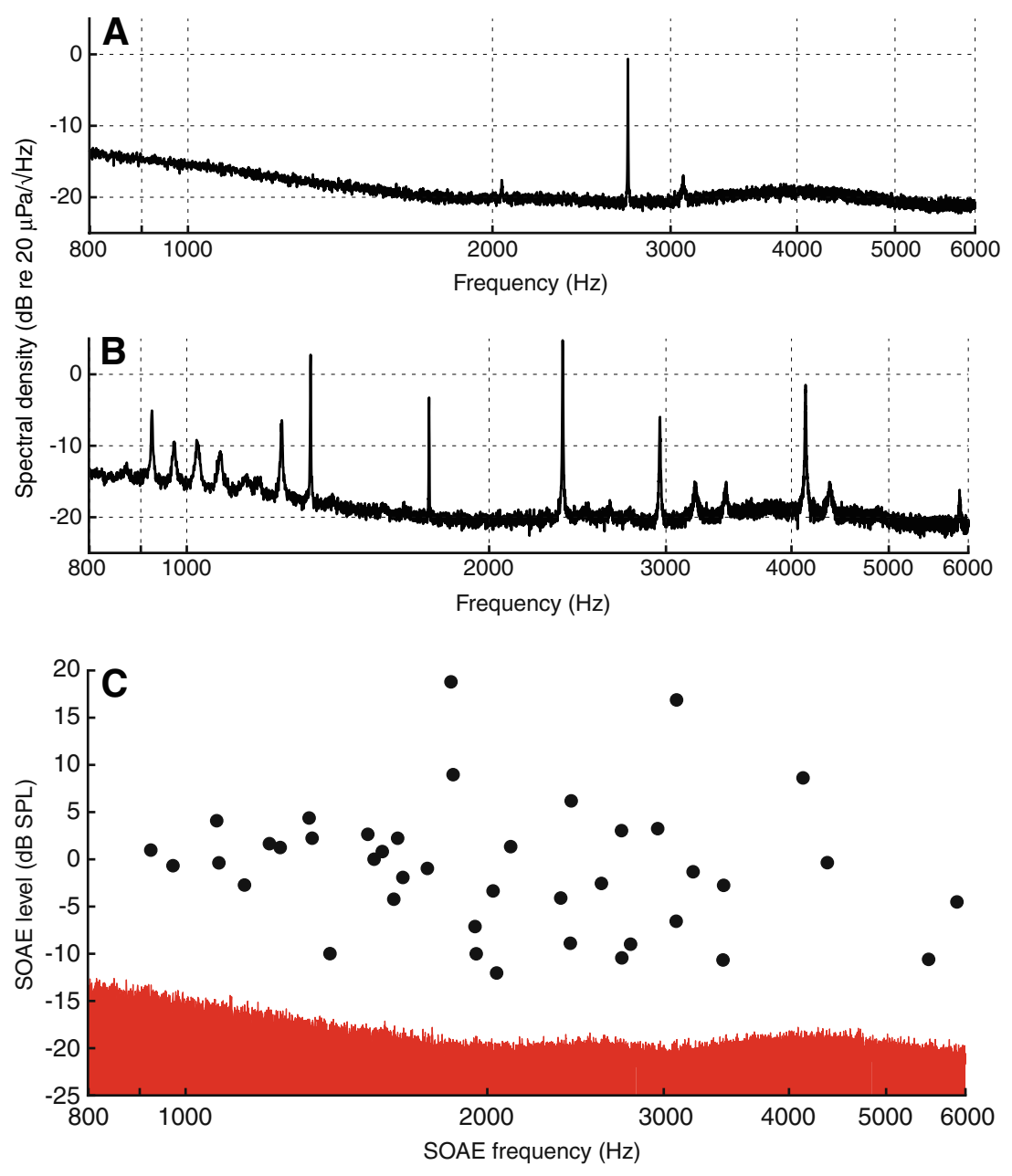

FIG. 1. A Spectrum of the spontaneous otoacoustic emission (SOAEs) recorded from subject LG. B Same for subject RZ. C Level and frequency of all 41 SOAE peaks from eight subjects, studied in this paper. The filled background shows the noise floor of the recording system in $1-\mathrm{Hz}$ bands.
Many SOAE peaks displayed a variation of the behavior shown in Figures 3 and 4. Some examples are shown in Figure 5. This subject was exceptional in that her ear emitted SOAEs at 15 frequencies, while in the other subjects a maximum of five SOAE peaks was detected. The peaks between 900 and $1,400 \mathrm{~Hz}$ are displayed in the figure. These peaks were closely spaced, that is, separated by about 50 $100 \mathrm{~Hz}$. The peaks below $1,100 \mathrm{~Hz}$ showed the "typical" behavior, similar to that in the previous figures: the SOAE frequency increased, and the SOAE amplitude decreased with both increasing and decreasing ear canal pressure. The peaks between 1,100 and $1,400 \mathrm{~Hz}$ showed a more complex behavior. For example, the peak between 1,200 and $1,250 \mathrm{~Hz}$ became a tall narrow peak at the most extreme pressures -200 and $+200 \mathrm{daPa}$. The peak near $1,300 \mathrm{~Hz}$ showed large jumps in amplitude and frequency, as illustrated in Figure 6. For small pressure deviations between -100 and $+100 \mathrm{daPa}$, this peak behaves similar to that illustrated in Figures 3 and 4 . The large jumps occurred for more extreme pressure values (greater than or equal to $+150 \mathrm{daPa}$ and less than or equal to-150 daPa).

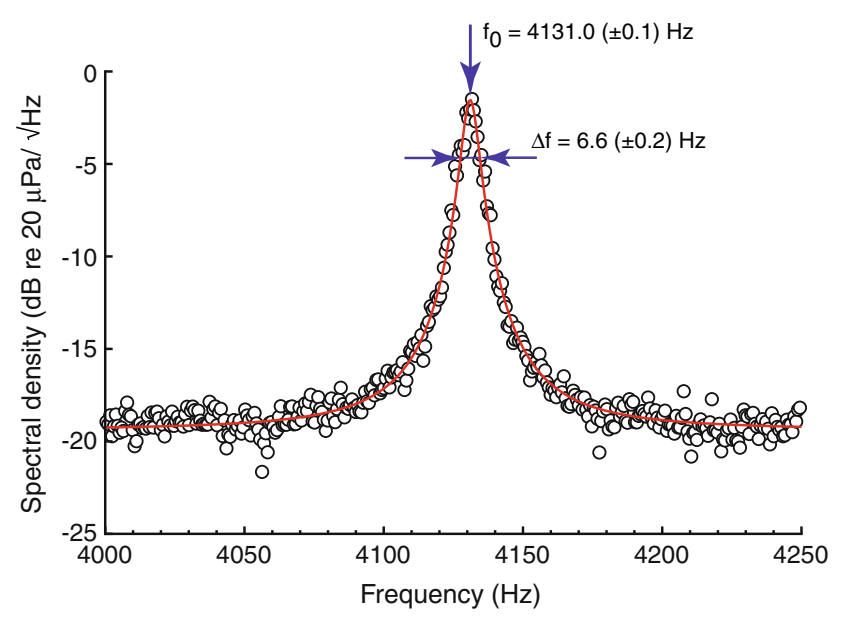

FIG. 2. Zoomed spectrum containing a single SOAE peak in subject RZ. The solid line is a Lorentzian curve (Eq. 15) fitted to the spectrum. The resulting fit parameters are: center frequency $f_{0}=$ $4131.0 \mathrm{~Hz}$, peak width at $3 \mathrm{~dB}$ below the maximum $\Delta f=6.6 \mathrm{~Hz}$, and the peak height re $-1.6 \mathrm{~dB}$ re $20 \mu \mathrm{Pa} / \sqrt{\mathrm{Hz}}$. The emission sound level corresponds to the area enclosed by the peak which corresponds to $8.6 \mathrm{~dB}$ SPL. 
A

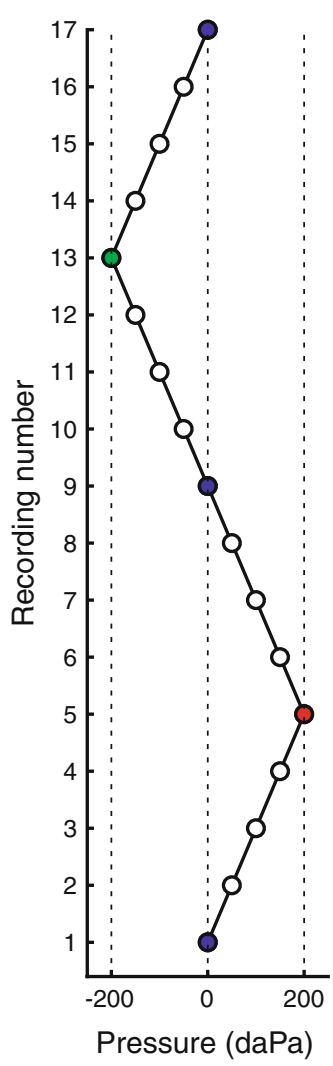

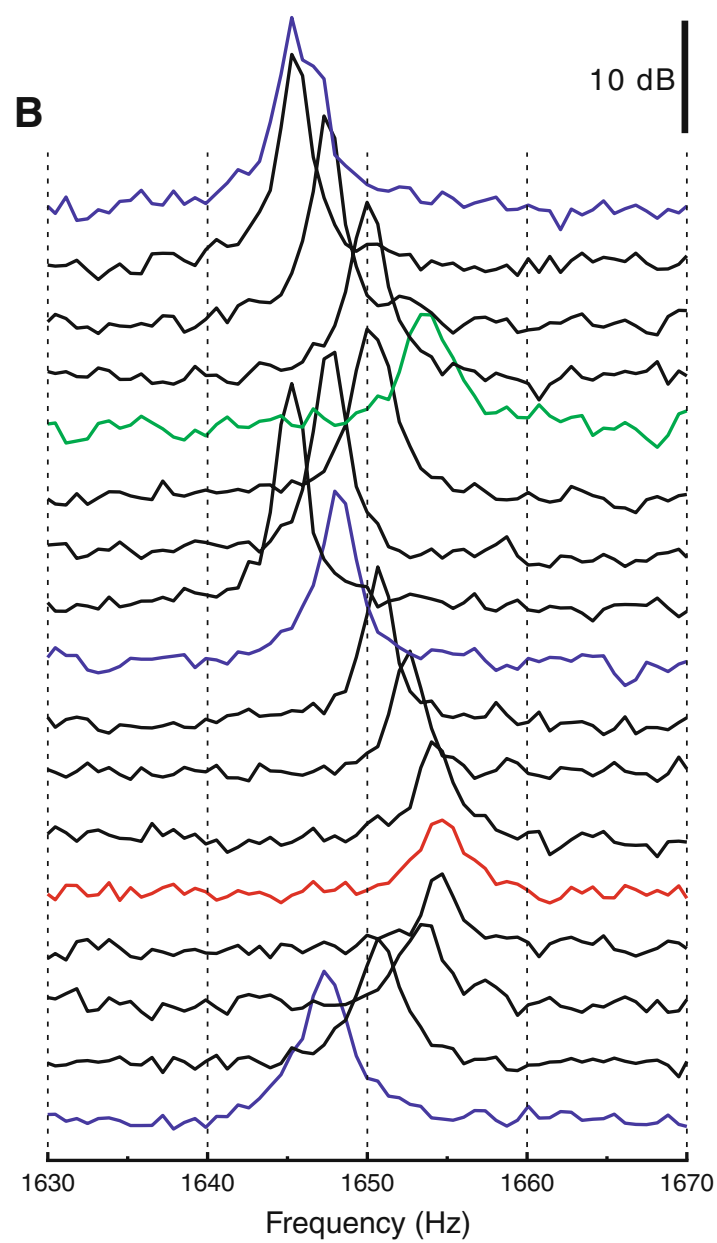

FIG. 3. Waterfall display of the relation between static ear canal pressure and the SOAE spectrum. The left panel shows the 17 static pressure settings at which 17 successive SOAE recordings were made. The right panel shows zoomed spectra for subject $\mathrm{KH}$ (shifted by $5 \mathrm{~dB}$ ).
Despite the irregular behavior of the SOAE amplitude and frequency, the relation between the peak height and width was regular, and followed the relation

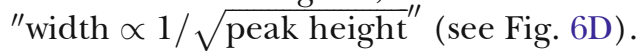

Figure 7 displays the outcome of the principal component analysis. Panel (A) displays the first and the second principal component $\mathcal{F}_{1}$ and $\mathcal{F}_{2}$ for frequency. The relative contribution of these components to the behavior of individual SOAE peaks was determined by minimizing the least-squares sum in Eq. 17. Recall that we here normalized each principal component such that its value at -200 daPa equalled +1 . Hence, the factors $b_{1}$ and $b_{2}$ are in Hertz and reflect frequency shifts at $-200 \mathrm{daPa}$. Figure $7 \mathrm{~B}$ displays these factors against SOAE frequency. This figure contains results for the 25 SOAE peaks included in the principal component analysis. In addition, these were also computed for the remaining 15 peaks for which only part of the data was available. This resulted in 15 additional data points in Figure 7B (and D).

The first principal component was approximately a linear curve and the corresponding factor strength $\left(b_{1}\right)$ was on average $0.2 \mathrm{~Hz}$ (s.d. $5.0 \mathrm{~Hz}$ ). The factor was not significantly correlated with SOAE frequency. The second principal component reflects the behavior typical for many SOAE peaks (see, e.g., Fig. 4A): both with increasing and decreasing pressure, the frequency increases. The corresponding factor strength $\left(b_{2}\right)$ was positive for 35 of the 40 SOAE peaks, with average factor strength $4.3 \mathrm{~Hz}$ (s.d. $10.0 \mathrm{~Hz})$. The second factor was correlated with SOAE frequency $(R=-0.48, p<0.01)$. Linear regression showed that the frequency shift tended to be smaller for SOAEs with a larger frequency, where the regression slope was $-4.4( \pm 1.3) \mathrm{Hz} /$ oct. The first two principal components were found to describe $80 \%$ of the variance of the SOAE frequency shifts.

The two outliers with $b_{2}<-20 \mathrm{~Hz}$ where excluded from this correlation/regression analysis, as the corresponding SOAE peaks were displaying negative frequency jumps. One of these peaks is illustrated in Figure 6.

A similar analysis was also conducted for the amplitude data, see Figure 7C and D. The first two components $\mathcal{A}_{1}$ and $\mathcal{A}_{2}$ were normalized to an amplitude shift equal to -1 at $-200 \mathrm{daPa}$. Thus, the factor strengths $a_{1}$ and $a_{2}$ are represented in $\mathrm{dB}$ and 

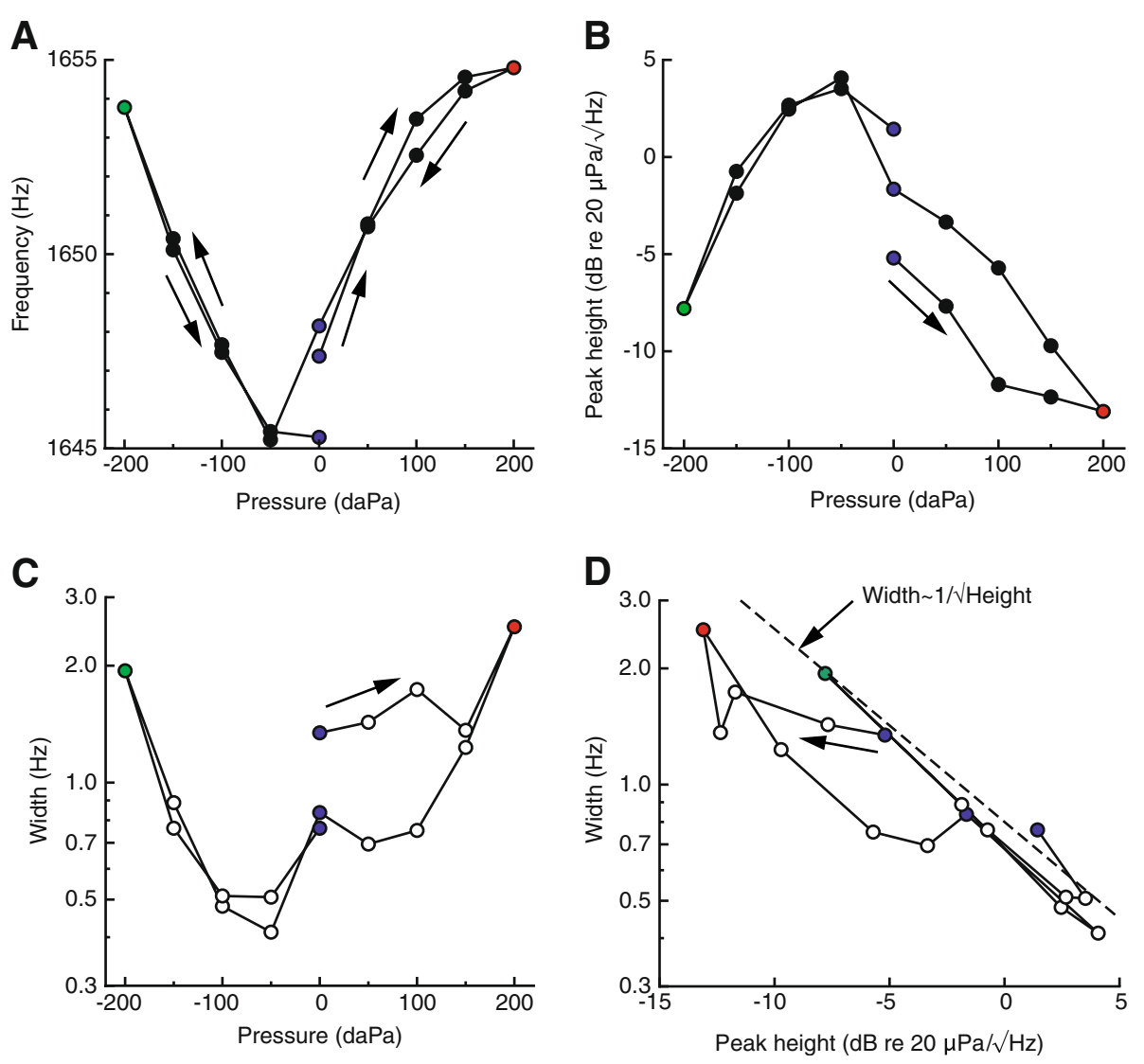

FIG. 4. Relation between SOAE characteristics and ear canal pressure for the peak in Figure 3. The peak height, center frequency and width were determined by fitting a Lorentzian curve to the frequency spectrum in Figure 3. A Frequency vs. pressure. B Height vs. pressure. C Width vs. pressure. D Width vs. height. The arrows show the measurement sequence. reflect the level shift at $-200 \mathrm{daPa}$. The first factor $a_{1}$ was negative for 34 out of $40 \mathrm{SOAE}$ peaks. Its average value was $-2.5 \mathrm{~dB}$ (s.d. $2.5 \mathrm{~dB}$ ). The factor was positively correlated with SOAE frequency $(R=0.46$, $p<0.01)$. The slope of a linear regression curve was +1.6 $( \pm 0.5) \mathrm{dB} /$ oct. In other words, the downward shift of SOAE level was less pronounced for SOAEs with a larger frequency. The factor strength $a_{2}$ of the second principal component was negative for 31 of 40 peaks, was on average $-1.1 \mathrm{~dB}$ (s.d. $3.5 \mathrm{~dB}$ ) and was not significantly correlated with SOAE frequency.

The principal component analysis was also conducted by combining the amplitude and frequency data in a single $(17+17) \times 25$ matrix. The first principal component that resulted from this combined analysis was similar to a combination of the second frequency component and the first amplitude component in Figure 7 (closed symbols). The second component of the combined analysis corresponded to the second frequency component and the first amplitude component (open symbols in Fig. 7).

Figure 8 shows the relation between peak width and peak height. As is also seen in Figures 4 and 6, the width of an SOAE peak is proportional to $1 / \sqrt{\text { height }}$. The relation between peak width and height also allows for the estimation of the average noise level $S_{\xi}$ in the equation of motion Eq. 12: -65.0 (s.d. 6.3) dB re $20 \mu \mathrm{Pa} / \sqrt{\mathrm{Hz}}$ (see also the inset of Fig. 8).

\section{DISCUSSION}

We systematically measured SOAEs at various ear canal pressures. Static ear canal pressure changed the properties of SOAEs. The most characteristic behavior was an increase in SOAE frequency and a decrease in SOAE amplitude with both increasing and decreasing static pressure. However, variation on this behavior existed, and the reverse effect was also observed. A remarkable finding is that the largest SOAE amplitude and the smallest SOAE frequency were often found at a slightly negative static pressure $(-50 \mathrm{daPa})$. These results are in general agreement with earlier reports on the effect of pressure on otoacoustic emission parameters (Schloth and Zwicker 1983; Hauser et al. 1993; Wada et al. 1995; Avan et al. 2000; Hof et al. 2005).

We applied principal component analysis (PCA) to provide a comprehensive description of the effect of pressure. A PCA identifies behavior that is common across subjects and across the emission peaks within subjects. The analysis was performed on the 25 emissions peaks (across the eight subjects) for which amplitude and frequency values were available for all 17 settings of the static ear canal pressure. The changes of SOAE frequency and amplitude are to a large extent described by the first two principal components. A linear combi- 
A

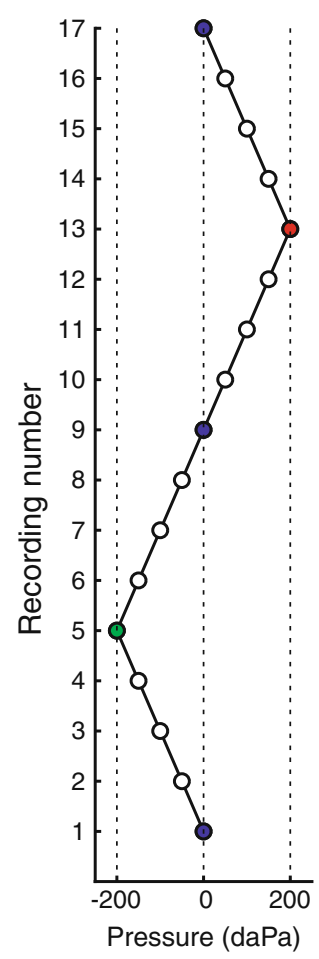

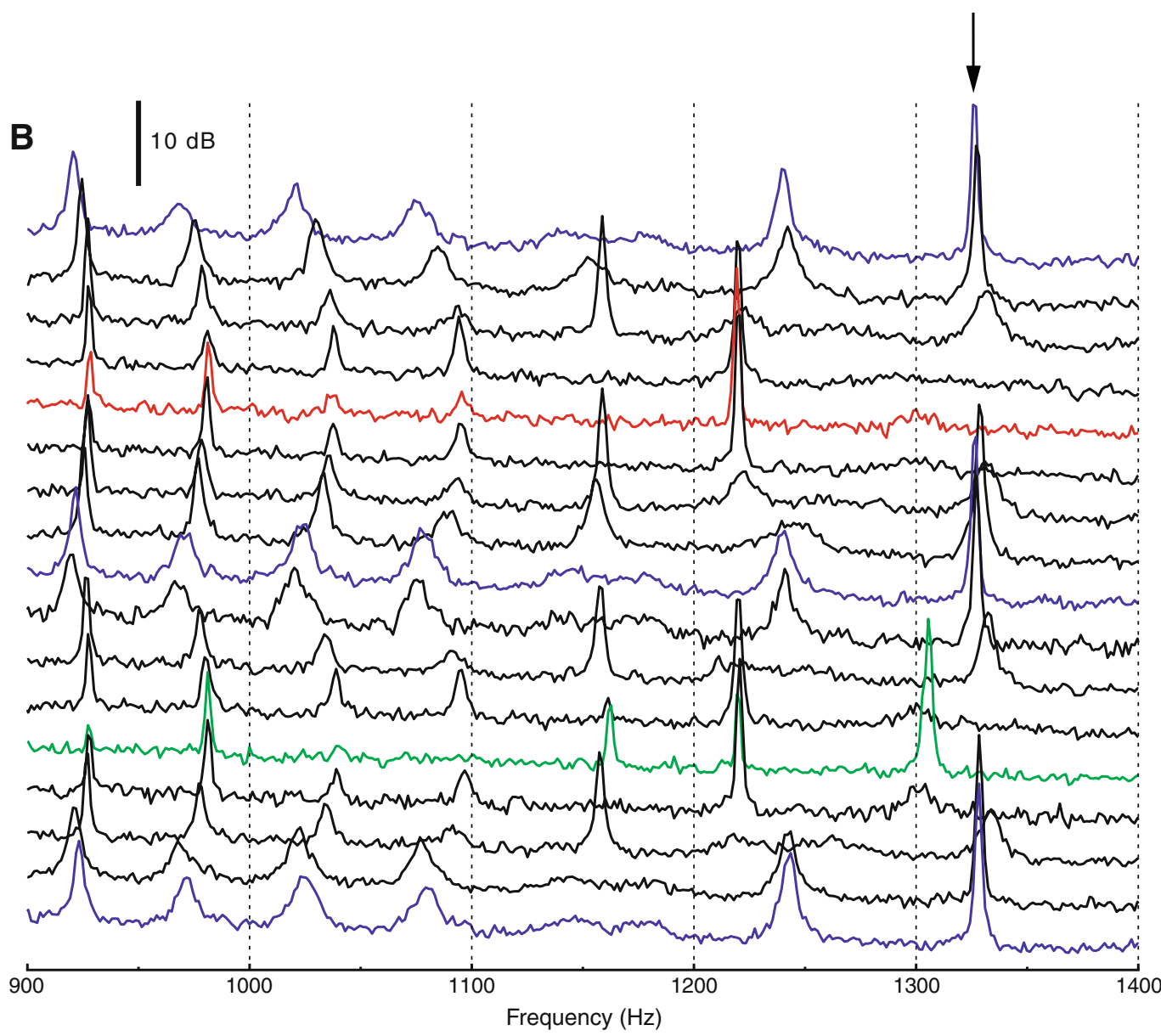

FIG. 5. Waterfall display of the relation between static ear canal pressure and the SOAE spectrum for subject RZ. The format is identical to that in Figure 3.

nation of the first two components of frequency described $80 \%$ of the frequency variance. The first two amplitude components described $74 \%$ of the amplitude variance.

The amplitude and frequency principal components presented in Figure 7 (panels [A] and [C]) are computed independently of each other. However, a PCA that combined amplitude and frequency data in a single analysis resulted in two principal components that were similar to the combination $\mathcal{F}_{1} / \mathcal{A}_{2}$ and $\mathcal{F}_{2} /$ $\mathcal{A}_{1}$. This suggests a relation between these amplitudefrequency pairs. Consequently, the pairs $\mathcal{F}_{1} / \mathcal{A}_{2}$ and $\mathcal{F}_{2} / \mathcal{A}_{1}$ are indicated with corresponding symbols in panels $[\mathrm{A}]$ and $[\mathrm{C}]$ of Figure 7.

Some considerations are relevant for the interpretation of the PCA. Firstly, an individual SOAE peak is traced over 17 pressure settings. Thus, peaks that have similar frequency in the 17 subsequent recording are assumed to correspond to "the same" emission component. This assumption is reasonable in peaks that shift only by a small amount between subsequent pressure settings. An example of such a peak is illustrated in Figure 4. However, in some cases, SOAE peaks shift over a large $(>10 \mathrm{~Hz})$ interval in subse- quent recordings, and it is unclear whether two peaks are "the same". An example of large frequency jumps is shown in Figure 6A. As described in the "Material and methods" section, all peaks that showed frequency jumps exceeding $10 \mathrm{~Hz}$ were excluded. Consequently, the PCA is very unlikely to be confounded by improper labeling of SOAE peaks as being "the same".

A second aspect that influences the PCA, is the inclusion of multiple peaks from the same subject. For example, subject RZ (see also Figs. 5 and 6) contributed eight peaks to the PCA, and one other subject contributed only one. Hence, the outcome may be biased to the behavior in those subjects that contribute several peaks.

The second frequency component and the first amplitude component describe the basic effect of pressure on SOAEs. Both components are indicated with closed symbols in Figure 7A and C, respectively. These two components are very similar to the effects of pressure on SOAEs as described by Schloth and Zwicker (1983) and others.

Interestingly, the first principal component for frequency is an approximately linear curve (open 

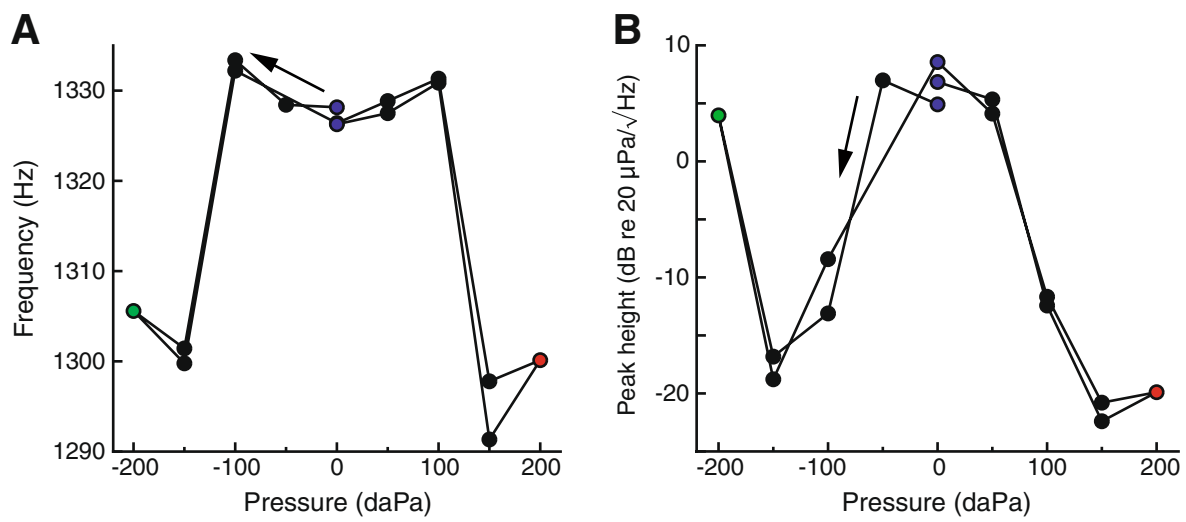

FIG. 6. Relation between SOAE characteristics and ear canal pressure for the peak in Figure 5 that is marked with an arrow. The format of this figure is identical to that of Figure 4.
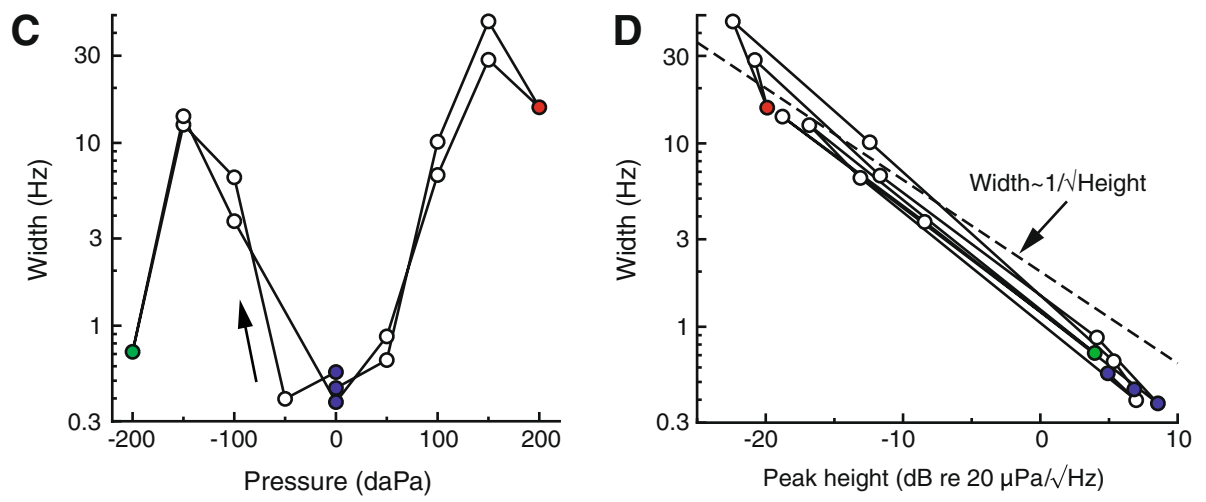

symbols in Fig. 7A). This is an example of the biasing effect that is described above: one subject who contributed four peaks to the PCA, showed such a linear relation for all SOAE peaks. Apparently, this subject biased the outcome of the PCA to have the approximately linear component as the first principle component.

Rather than concentrating on the ranking of components, it is more insightful to consider the factor strengths that describe to what extent each component contributes to the frequency shifts and amplitude changes of individual peaks. As is shown in Figure $7 \mathrm{~B}$, the factor strength of the second frequency component is positive for 34 of the 40 SOAE peaks. Given the V-shape of the second principal component (Fig. 7A), this corresponds to an increase of SOAE frequency with either increasing or decreasing static pressure. In contrast, the factor of the first amplitude component was negative for 34 of 40 SOAE peaks (Fig. 7D). With the inversed V-shape of the corresponding component (Fig. 7C), this indicates a decrease of the amplitude with changing static pressure for most of the SOAE peaks. The factor strength of both these components correlates with SOAE frequency, which shows that for low-frequency SOAEs the increase of frequency and the decrease of amplitude, tends to be larger than for high-frequency SOAEs. This is consistent with the trends observed by other authors (e.g., Hauser et al. 1993) and for other experimental manipulations, such as body tilt (De
Kleine et al. 2000; Voss et al. 2010), evoking the stapedius reflex (Avan et al. 2000) and increasing the intra-cranial fluid pressure (Büki et al. 2000). All these manipulations are believed to result in a change of the stapes impedance, either due to inner ear fluid pressure increase (tilt, intra-cranial fluid pressure), or by tension of the stapedius muscle. Apparently, all these manipulations mostly influence the impedance at lower frequencies.

In contrast, the factors of the first frequency component and the second amplitude component scatter around 0 and do not show a correlation with SOAE frequency (open symbols in Fig. 7B and D). These two principal components do not show a clear symmetry with respect to the static pressure $p=-25 \mathrm{daPa}$. Rather, they represent an asymmetry in the effect of positive versus negative ear canal pressure. Apparently, this asymmetry is not systematically varying along the cochlear partition.

Static ear canal pressure changes the properties of the middle ear (see e.g., Lee and Rosowski 2001; Gea et al. 2010; Homma et al. 2010). The effects of static ear canal pressure on SOAEs presumably result from the changes of the middle ear. Both positive and negative pressures result in stretching of the tympanic membrane. This may change the characteristics of outward transmission through the middle ear. Also, it may change the impedance of the oval window, as seen from within the cochlea. In other words, static ear canal pressure may result in a change of the 

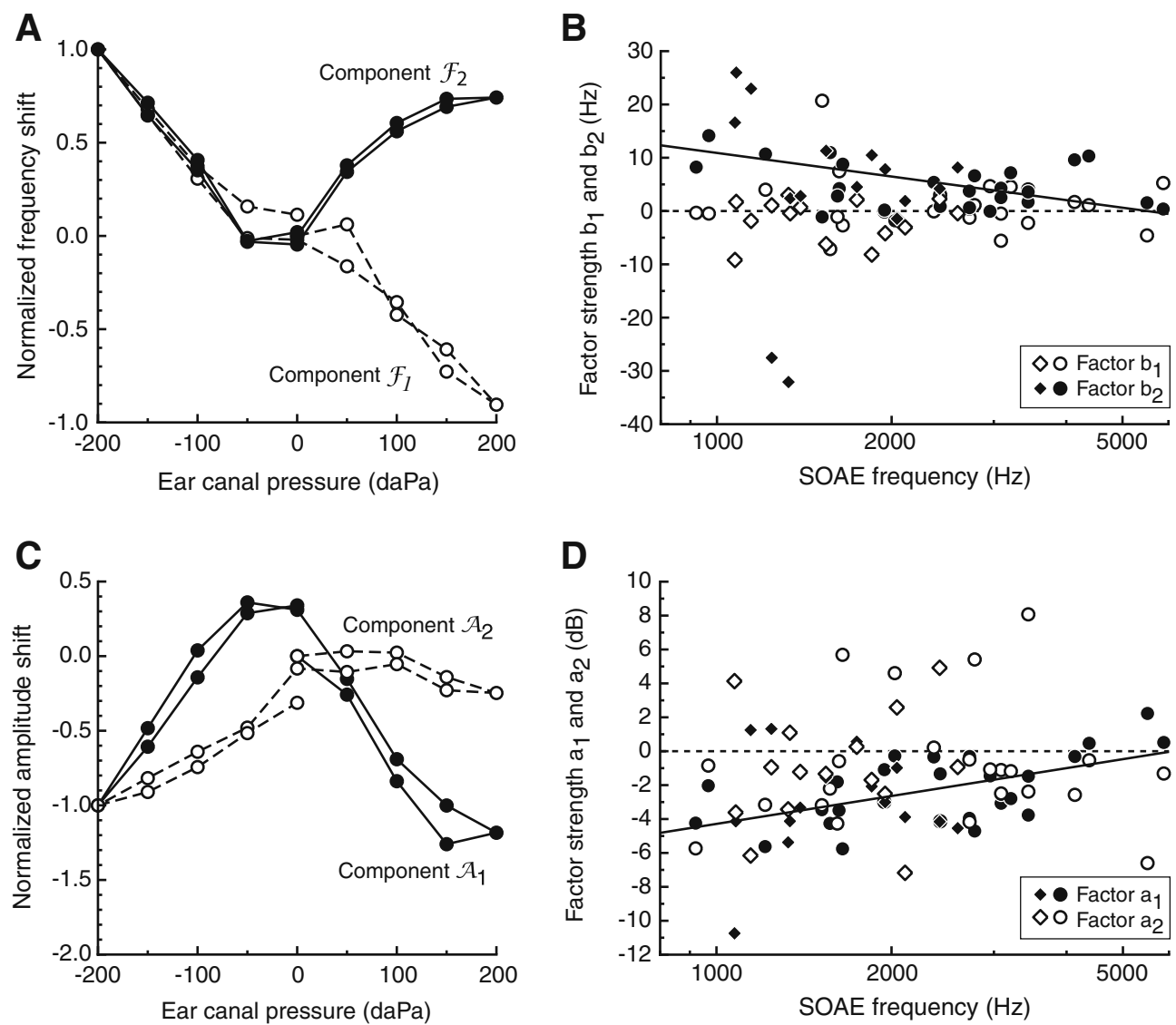

FIG. 7. Principal component analysis (PCA) of the behavior of SOAE frequency and amplitude. A The first two principal components for SOAE frequency. These components were normalized to +1 at $-200 \mathrm{daPa}$. Open symbols: the first component. Closed symbols: the second component. B Factor strength of the contribution of the first two components to the behavior of individual SOAE peaks. A positive factor indicates a positive frequency shift [in $\mathrm{Hz}]$ at -200 daPa. Open symbols: the first component. Closed symbols: the second component. The circles show results for the 25 emission peaks included in the PCA analysis. The squares corresponded to the 15 remaining peaks, which could not be included in the PCA analysis, but for which the factor analysis could be applied (see main text). The solid line is a regression curve through the closed data points, where the two outliers below $-20 \mathrm{~Hz}$ were excluded (see

impedance load on the emission generators in the cochlea.

In addition to a direct effect of the changing stapes impedance on the emission generator in the ear, also the interaction between multiple emissions may contribute to the observed effects. Individual SOAE peaks in an emission recording are not independent. Their amplitudes may be correlated, as is clear from their correlated amplitude fluctuations (Burns et al. 1984; Van Dijk et al. 1994) and from the correlated relaxation behavior in response to an external stimulus (Jones et al. 1986; Murphy et al. 1995b). The complicated behavior of a number of peaks displayed in Figures 5 and 6 may be based on interactions between emission peaks. text). The slope of this curve is $-4.4( \pm 1.3) \mathrm{Hz} /$ oct. C The first two principal components of emission amplitude. Closed symbols: first component. Open symbols: second component. The components were normalized to an amplitude shift -1 at -200 daPa. The symbol choice reflects the fact that components with the closed symbols in panel $\mathbf{A}$ and $\mathbf{C}$ together describe one aspect of SOAE behavior. Similarly, the open symbols describe another aspect (see the main text for the combined PCA for amplitude and frequency). D Factor strength of the contribution of the first two components to SOAE amplitude behavior. A negative value in $\mathrm{dB}$ corresponds to an amplitude decrease at $-200 \mathrm{daPa}$. The solid line is a regression curve through the closed symbols with slope $+1.6( \pm 0.5) \mathrm{dB} /$ oct. The symbols have the same meaning as in panel $\mathbf{B}$.

A change in the middle ear could influence the otoacoustic emission amplitude in (at least) two ways. Either the middle ear transmission from the inner ear to the ear canal is affected, or the intra-cochlear emission source signal is changed, for example due to a changed oval window impedance. In order to distinguish between these two possibilities, we investigated the relation between peak width and height of SOAE peaks. As was described in the "Theory" section, the Rayleigh oscillator model predicts the relation between the peak width and height for both possibilities (see Eqs. 13 and 14).

For SOAEs, it was clear that peak width was

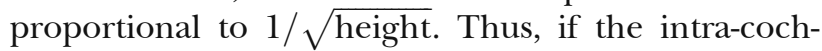
lear noise that disturbs the SOAE generator is broad- 


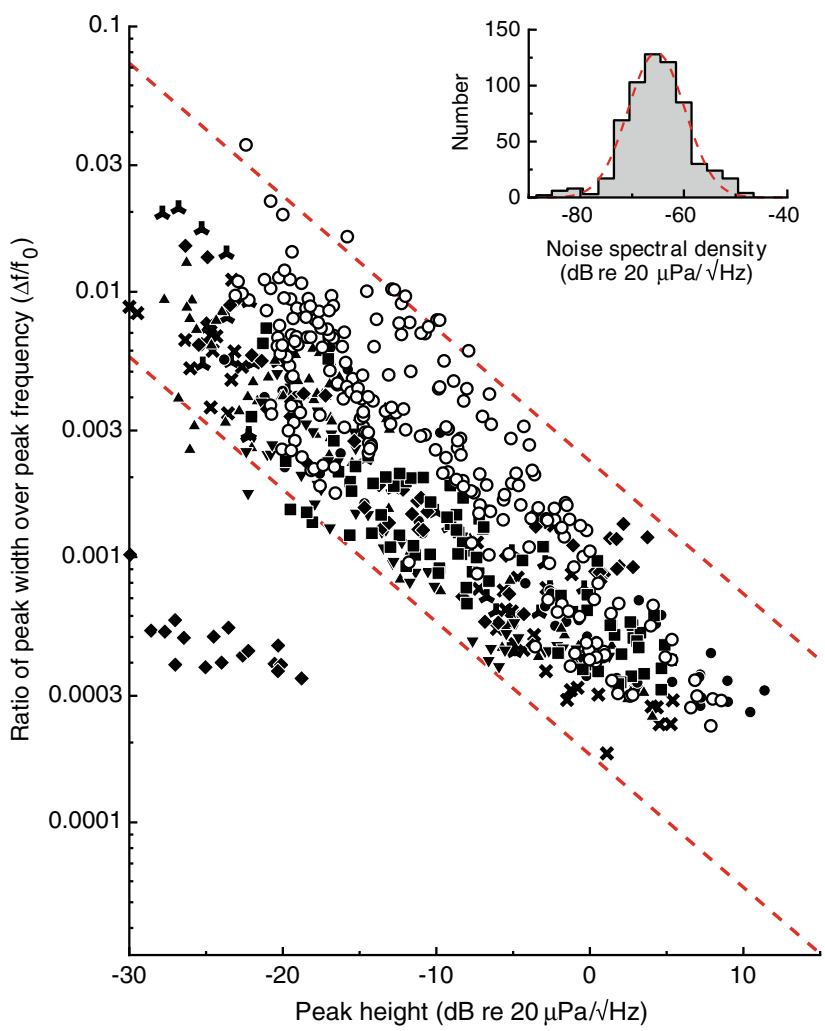

FIG. 8. Relative peak width versus peak height. Each symbol type corresponds to a subject. Each subject contributes data for several SOAE peaks to this figure. Each SOAE peak is represented by several data points, corresponding to the various static pressure settings, respectively. Eq. 12 relates the level, the center frequency and the width of a peak to the level of internal noise $\left(S_{\xi}\right)$. The internal noise level was computed for each data point in this scatter plot. The inset shows a histogram of the noise estimates. The dashed curve in the inset is a fit to the histogram. It peaks at -65.0 (s.d. 6.3) $\mathrm{dB}$ re $20 \mu \mathrm{Pa} / \sqrt{\mathrm{Hz}}$.

band Gaussian noise, and the spectral density of this noise is constant, the changes in SOAE amplitude must be related to changes of the intra-cochlear source signal.

Interestingly, most individual SOAE peaks do not entirely follow the proportionality relation $\Delta \omega \propto 1 / \sqrt{S_{x}\left(\omega_{0}\right)}$ (Eq. 13). Both examples given in the paper (Figs. 4D and 6D) show deviations from the dashed diagonal. The dashed curve is based on the Rayleigh model. The curve applies when we assume (1) the transmission ratio of the middle ear to be fixed and (2) the internal noise to be independent of static ear canal pressure. The deviations from the dashed curve suggest that these assumptions may be only approximately valid. Also, interaction between SOAEs, which are not part of the model, may explain the deviations. Finally, the relation between peak height and width (i.e. Fig. 4D) suggests some hystersis in these phenomena. Thus, the Rayleigh model describes an important part of the relation between emission peak height and width, but several aspects of the data cannot be captured by this simple model.
In the model description, we assumed the noise to be broadband Gaussian noise. Then, the oscillator phase freely diffuses, which results in broadening of the oscillation spectrum. The Lorentzian shape of SOAE peaks is in excellent agreement with the free phase diffusion predicted by the Rayleigh model (see Fig. 2). It is possible that the Gaussian noise in the model represents thermal noise in the cochlea. However, periodic frequency fluctuations that are in phase with heartbeat, are known to be a significant component of the frequency and amplitude fluctuations of SOAEs (Bell 1992; Long and Talmadge 1997). The primary lines of evidence for these fluctuations are side bands to the main peak of strong SOAE, at a distance that relates to the heart rate. We did not observe sidebands in the SOAE spectra. As was pointed out by Long and Talmadge, these fluctuations can be detected for strong otoacoustic emissions. In contrast, the majority of the emission peaks studied in the current paper have levels below 5 dB SPL (see Fig. 1). Possibly, we did not see clear indications of heartbeat modulation of SOAEs, either because most of the emissions were relatively weak, or because we made relatively short recordings $(1 \mathrm{~min})$ and did not directly correlate the emission signals with a recording of blood flow.

It is likely that both heartbeat and thermal noise affect the stability of the emission signal. In the case of weak emissions, it may be that the phase diffusion that results from thermal noise dominates over the periodic fluctuations that result from periodic blood flow. Then, the main SOAE peak and its small sidebands would smear out and result in a single broad spectral peak. With the recordings in the current paper, it is impossible to identify the relative contribution of periodic heartbeat and random phase diffusion to the frequency instability of SOAEs. In order to further investigate the relative contribution of both noise sources, it is necessary to make long recordings (Long and Talmadge used $5 \mathrm{~min}$ ) of both the SOAE signal and the heartbeat signal. Possibly, such recordings made at a range of static ear canal pressures may clarify to what extend blood flow and thermal noise each affect the intra-cochlear source signal and the transmission of the emission signal through the middle ear. If heartbeat turns out to be the dominant component of the frequency instability, it remains unexplained how that peak width is proportional to $1 / \sqrt{\text { height }}$.

Under the assumption, that wideband Gaussian noise is the primary factor that determined the width of the SOAE peaks in the spectra, we estimated the noise level to be $-65.0 \mathrm{~dB}$ re $20 \mu \mathrm{Pa} / \sqrt{\mathrm{Hz}}$ (see Fig. 8, inset). This number is expressed as a sound pressure level at the eardrum. Within one equivalent rectangular band, which has a width of about $250 \mathrm{~Hz}$ near $2 \mathrm{kHz}$ (Glasberg and Moore 1990), this corresponds to $-65.0+10 \times \log _{10}(250)=-41 \mathrm{~dB}$ SPL. This estimate is four orders of magnitude below the threshold 
of hearing (about $0 \mathrm{~dB}$ SPL). Consequently, it is unlikely that the noise that destabilizes the frequency of SOAEs is a limiting factor of the sensitivity of the inner ear. At present, we are puzzled by the low level of the inherent noise of SOAEs, and have no clear interpretation of the functional significance of this noise level.

Although the Rayleigh oscillator model provides a model for the relation between emission peak height and width, it cannot account for the effect of static ear canal pressure on the SOAE frequency. A more comprehensive middle and inner ear model is presumably required for that. A leading hypothesis on the origin of SOAEs is that they correspond to a standing wave in the cochlea. This standing wave may build up between the stapes and a location near the characteristic place of the emission frequency (Zweig and Shera 1995; Shera 2003). In this hypothesis, a retrograde cochlear wave that travels from the characteristic place in the cochlea to the stapes, is partly reflected at the stapes and partly transmitted through to middle ear into the ear canal. Hence, it results in a detectable emission signal in the ear canal. In response to increasing or decreasing static ear canal pressure, the frequency of an SOAE peak most often increased. This frequency shift is consistent with the standing-wave model of SOAE generation, if we assume that a change of the static pressure increases the mechanical stiffness of the oval window. Stiffening of the oval window will reduce the phase shift of the reflection of the retrograde wave. This reduces the roundtrip travelling time between the stapes and the characteristic place in the cochlea, and the standing wave frequency will increase. Thus, in contrast to the simple oscillator model presented here, a more comprehensive standing wave mode of SOAE generation is likely to account for the effect of ear canal pressure on the emission frequency.

Finally, the fact that Rayleigh (and Van der Pol) oscillator models capture many aspects of SOAEs may not be considered evidence against a standing-wave model of emission generation. It is conceivable that a single standing-wave SOAE behaves similarly to a Rayleigh oscillator. However, several aspects of the data presented in this paper, such as interaction between multiple SOAE peaks and the deviation of peaks from the simple model behavior require a more comprehensive cochlear model.

\section{CONCLUSIONS}

In conclusion, we showed that a change of static ear canal pressure affects the amplitude, the frequency and the width of SOAEs. The relation between peak height and width was modeled with a Rayleigh oscillator model in which noise slightly destabilizes the emission frequency. This noise level is assumed to be independent of the static ear canal pressure. The relation between SOAE peak height and width is consistent with a direct effect of static ear canal pressure on the intra-cochlear emission source signal amplitude. Thus, the ear canal static pressure directly affects the intra-cochlear mechanics.

\section{ACKNOWLEDGEMENT}

We thank Dave Langers for suggesting the PCA. This work is supported by the Heinsius Houbolt Foundation, the Huizinga Foundation, and the Netherlands Organization for Scientific Research (NWO).

\section{OPEN ACCESS}

This article is distributed under the terms of the Creative Commons Attribution Noncommercial License which permits any noncommercial use, distribution, and reproduction in any medium, provided the original author(s) and source are credited.

\section{APPENDIX}

This appendix derives an expression for the spectral density (the spectrum) of a Rayleigh oscillator that is exposed to wideband Gaussian noise. The derivation closely follows and partly replicates that given by Stratonovich (1963). First, we layout the behavior of a Rayleigh oscillator in absence of a disturbing noise. Second, we will derive an expression for the spectrum of a Rayleigh oscillator that is exposed to weak wideband Gaussian noise. As is shown, the spectrum consists of a peak that broadens as a result of the disturbing noise.

In order to derive the behavior of a Rayleigh oscillator, it is convenient to rewrite the second-order Rayleigh differential equation Eq. 2 as two first-order equations for the amplitude and phase of the oscillation. The amplitude and phase are defined by two equations, that relate them to the oscillation signal $x(t)$ and its time derived (e.g. the velocity) $\dot{x}(t)$ :

$$
\begin{gathered}
x(t)=A \cos \left(\omega_{0} t+\phi\right) \\
\dot{x}(t)=-A \omega_{0} \sin \left(\omega_{0} t+\phi\right)
\end{gathered}
$$

In general, $A(t)$ and $\phi(t)$ are time-dependent, but will drop the explicit notation of time. The amplitude and phase follow directly from these two equations as

$$
A=\sqrt{x^{2}+\frac{\dot{x}^{2}}{\omega_{0}^{2}}}
$$




$$
\phi=-\arctan \frac{\dot{x}}{\omega_{0} x}-\omega_{0} t
$$

By differentiating these equations, we arrive at two first-order differential equation for the amplitude and the phase:

$$
\begin{gathered}
\dot{A}=\frac{\dot{x}}{\omega_{0}^{2} A}\left(\ddot{x}+\omega_{0}^{2} x\right) \\
\dot{\phi}=-\frac{x}{\omega_{0} A^{2}}\left(\ddot{x}+\omega_{0}^{2} x\right)
\end{gathered}
$$

These equations allow for straightforward substitution of the Rayleigh equation of motion Eq. 2. First, we consider the amplitude Eq. 23, which becomes

$$
\begin{aligned}
\dot{A}= & \frac{\dot{x}}{\omega_{0}^{2} A}\left[\varepsilon \omega_{0}\left\{1-\frac{4 \dot{x}^{2}}{3 \omega_{0}^{2} A_{0}^{2}}\right\} \dot{x}+\omega_{0}^{2} \xi(t)\right] \\
= & \frac{\varepsilon \dot{x}}{\omega_{0} A}-\frac{4 \varepsilon \dot{x}}{3 \omega_{0}^{3} A A_{0}^{2}}+\frac{\dot{x}}{A} \xi(t) \\
= & \varepsilon \omega_{0} A \sin ^{2} \Phi-\frac{4 \varepsilon A^{3} \omega_{0}}{3 A_{0}^{2}} \sin ^{4} \Phi-\omega_{0} \xi(t) \sin \Phi \\
= & \varepsilon \omega_{0} A\left(\frac{1}{2}-\frac{1}{2} \sin 2 \Phi\right)-\frac{4 \varepsilon A^{3} \omega_{0}}{3 A_{0}^{2}}\left(\frac{3}{8}-\frac{1}{2} \cos 2 \Phi+\frac{1}{8} \cos 4 \Phi\right) \\
& -\omega_{0} \xi(t) \sin \Phi
\end{aligned}
$$

where we introduced $\Phi=\omega_{0} t+\phi$ in order to simplify the notation. We will now consider the solution of this equation in absence of the disturbing noise, i.e., $\xi(t)=0$. Then, the equation can be simplified by assuming that the amplitude is a slowly varying function. Consequently, the terms in Eq. 25 can be replaced by their average across one period of the oscillation. The rapidly oscillating terms containing $\sin 2 \Phi, \cos 2 \Phi$, and $\cos 4 \Phi$, all average to 0 , and the equation simplifies to:

$$
\dot{A}=\frac{\varepsilon \omega_{0} A}{2}\left[1-\frac{A^{2}}{A_{0}^{2}}\right]
$$

A stable solution, with $\dot{A}=0$, is given by

$$
A(t)=A_{0}
$$

Equations 25 and 26 offer the opportunity to further explore the behavior of the oscillator amplitude. In the context of spontaneous otoacoustic emissions, this is relevant for describing their amplitude relaxation behavior (Schloth and Zwicker 1983; Murphy et al. 1995a) and their random amplitude fluctuations (Bialek and Wit 1984; Van Dijk and Wit 1990a). However, here we concentrate on the properties of spectral peaks in an emission spectrum. As is shown by
Stratonovich (1963), the amplitude fluctuations have a minor (second-order) effect on the oscillator spectrum, if the noise spectral density $S_{\xi}$ is relatively small, e.g., $S_{\xi}\left(\omega_{0}\right)<A_{0}^{2} / \omega_{0}$. Then, the spectrum of the oscillator signal is mostly determined by the phase fluctuations. Hence, since we are interested in the properties of the spectrum of the oscillator, we will not further explore amplitude fluctuations. For brevity, we will simply assume the amplitude to be stable (Eq. 27).

The behavior of the oscillator phase follows by substitution of the equation of motion (2) into the phase Eq. 24. Similar to the method that led to Eq. 25, we will assume slow fluctuation of the phase and will average across one period in order to eliminate rapidly oscillating terms. Then, the phase equation becomes

$$
\dot{\phi}=-\frac{\omega_{0}}{A_{0}} \xi(t) \cos \Phi
$$

Thus, averaging across one period of the oscillation only retains the term that contains the noise $\xi(t)$. In absence of this term, e.g., with $\xi(t)=0$, the phase is solved by

$$
\phi(t)=\phi_{0}
$$

where $\phi_{0}$ is an arbitrary constant. If the noise is nonzero, the phase will fluctuate, and the phase drift over a time interval $\tau$ can be obtained by integrating Eq. 28:

$$
\Delta \phi=-\int_{0}^{\tau} d t \frac{\omega_{0}}{A_{0}} \xi(t) \cos \Phi
$$

This integral is complicated by the presence of the factor $\cos \Phi$ on the right hand side. However, we will consider the case where $\xi$ is white Gaussian noise, with a constant spectral density $S_{\xi}$ and an autocorrelation function that is given by a Dirac delta function:

$$
R_{\xi}(t)=S_{\xi} \delta(t)
$$

Then, the statistical properties of the product $\xi(t)$ $\cos (\Phi)$ in the phase Eq. 28 are identical to that of a white noise $\zeta(t)$ with spectral density $S_{\zeta}=S_{\xi} / 2$. Consequently, the properties of the phase Eq. 28 are identical to that of the equation

$$
\dot{\phi}=-\frac{\omega_{0}}{A} \zeta(t),
$$

where the noise term $\zeta(t)$ has an autocorrelation function

$$
R_{\zeta}(t)=\frac{S_{\xi}}{2} \delta(t)
$$


The integral Eq. 30 is replaced by a simplified equation

$$
\Delta \phi=-\int_{0}^{\tau} d t \frac{\omega_{0}}{A_{0}} \zeta(t)
$$

Since $\zeta(t)$ is a Gaussian variable with zero mean, the same applies to the phase drift $\Delta \phi$. The variance of $\Delta \phi$ is

$$
\begin{aligned}
\left\langle(\Delta \phi)^{2}\right\rangle & =\frac{\omega_{0}^{2}}{A_{0}^{2}}\left\langle\int_{0}^{\tau} \int_{0}^{\tau} d t_{1} d t_{2} \zeta\left(t_{1}\right) \zeta\left(t_{2}\right)\right\rangle \\
& =\frac{\omega_{0}^{2} S_{\xi}}{2 A_{0}^{2}} \int_{0}^{\tau} d t_{1}=D \tau,
\end{aligned}
$$

where the autocorrelation function Eq. 33 was used and

$$
D=\omega_{0}^{2} S_{\xi} / 2 A_{0}^{2}
$$

is termed the phase diffusion constant. Thus, the variance of the phase drift increases linearly with $\tau$. In other words, the phase diffuses freely and for larger time intervals, the phase drift is more likely to be large.

Stratonovich (1963) derives the diffusional phase behavior for broadband Gaussian noise, which is slightly more general that the white Gaussian noise that was considered in the present paper. If the noise is broadband, its spectral density is not necessarily constant. As Stratonovich (1963), the factor $S_{\xi}$ in the above equation can be replaced the spectral density $S_{\xi}\left(\omega_{0}\right)$ at the oscillator frequency, and the diffusion constant is given by Eq. 10 in the main text.

The spectrum of the oscillator signal $x(t)$ can be obtained by first computing its autocorrelation function:

$$
\begin{aligned}
R_{x}(\tau) & \equiv\langle x(t) x(t+\tau)\rangle \\
& =A_{0}^{2}\left\langle\cos \left(\omega_{0} t+\phi(t)\right) \cos \left(\omega_{0}\{t+\tau\}+\phi(t+\tau)\right)\right\rangle \\
& =\frac{A_{0}^{2}}{2}\left\langle\cos \left[2 \omega_{0} t+\omega_{0} \tau+2 \phi_{0}+\delta \phi(t)+\delta \phi(t+\tau)\right]\right. \\
& \left.+\cos \left[\omega_{0} \tau+\delta \phi(t+\tau)-\delta \phi(t)\right]\right\rangle
\end{aligned}
$$

In this equation, the brackets $\langle\cdots\rangle$ represent an ensemble average. That is, the average is taken across realizations of the noise $\xi$. The constant $\phi_{0}$ is the phase at $t=0$, which will be uniformly distributed in the ensemble. Since the first term in the above equation contains the initial phase $\phi_{0}$ in the cosine function, its ensemble average will be zero. The function $\delta \phi(t)$ is the total phase drift since $t=0$. The second term in Eq. 37 contains the difference $\Delta \phi=\delta \phi(t+\tau)-\delta \phi(t)$. As we derived in the previous paragraph, this phase difference is a Gaussian variable with variance $D \tau$ (see Eq. 35). Hence, the ensemble average in the autocorrelation function can be computed by substitution of a Gaussian probability density with variance $D \tau$ :

$$
\begin{aligned}
R_{x}(\tau) & =\frac{A_{0}^{2}}{2}<\cos \left[\omega_{0} \tau+\Delta \phi\right]> \\
& =\frac{A_{0}^{2}}{2} \int_{-\infty}^{\infty} d \alpha \frac{e^{-\alpha^{2} / 2 D \tau}}{\sqrt{2 \pi D \tau}} \cos \left[\omega_{0} \tau+\alpha\right]
\end{aligned}
$$

where the integration variable $\alpha$ now represents phase drift. Note that $\cos \left[\omega_{0} \tau+\alpha\right]=\cos \omega_{0} \tau \cos \alpha-\sin \omega_{0} \tau$ $\sin \alpha$. The term in the integral that contains $\sin \alpha$ integrates to 0 , and consequently the autocorrelation function becomes

$$
\begin{aligned}
R_{x}(\tau) & =\frac{A_{0}^{2}}{2} \int_{-\infty}^{\infty} d \alpha \cos \omega_{0} \tau \frac{e^{-\alpha^{2} / 2 D \tau}}{\sqrt{2 \pi D \tau}} \cos \alpha \\
& =\frac{A_{0}^{2}}{2} e^{-\frac{1}{2} D \tau} \cos \omega_{0} \tau
\end{aligned}
$$

The spectral density of the oscillator signal is defined as the Fourier transform of the autocorrelation function:

$$
\begin{aligned}
S_{x}(\omega) & =\int_{-\infty}^{\infty} e^{i \omega \tau} R_{x}(\tau) d \tau \\
& =\frac{A_{0}^{2} D}{2} \frac{\omega^{2}+\omega_{0}^{2}+\frac{1}{4} D^{2}}{\left(\omega^{2}-\omega_{0}^{2}-\frac{1}{4} D^{2}\right)^{2}+D^{2} \omega^{2}} \\
& \approx \frac{A_{0}^{2}}{4} \frac{D}{\left(\omega-\omega_{0}\right)^{2}+\frac{1}{4} D^{2}},
\end{aligned}
$$

where the last approximation applies if the noise is weak and $D \ll \omega_{0}$. The spectrum $S_{x}$ contains a peak at $\omega=\omega_{0}$, that has a full width at half maximum (FWHM) that equals the phase diffusion constant: $\Delta \omega=D$. From Eq. 36 it follows that this width increases if the noise spectral density $S_{\xi}$ increases. Also, the width decreases if the oscillation amplitude $A_{0}$ increases. Hence, the width is a measure of the signal-to-noise ratio of the oscillator, where signal refers to the oscillation amplitude and noise refers to the noise that disturbs the oscillator.

\section{REFERENCES}

Avan P, Büki B, MaAt B, Dordain M, Wit HP (2000) Middle ear influence on otoacoustic emissions. I: noninvasive investigation of the human transmission apparatus and comparison with model results. Hear Res 140:189-201

Bell A (1992) Circadian and menstrual rhythms in frequency variations of spontaneous otoacoustic emissions from human ears. Hear Res 58:91-100

Bialek W, Wit HP (1984) Quantum limits to oscillator stability: theory and experiments on acoustic emissions from the human ear. Phys Lett 104A:173-178

Büki B, Chomicki A, Dordain M, Lemaire J-J, Wit HP, Chazal J, Avan P (2000) Middle-ear influence on otoacoustic emissions. II: 
contributions of posture and intracranial pressure. Hear Res 140:202-211

Burns EM, Strickland EA, Tubis A, Jones K (1984) Interactions among spontaneous otoacoustic emissions. I. Distortion products and linked emissions. Hear Res 16:271-278

De Kleine E, Wit H, Van Dijk P, Avan P (2000) The behavior of spontaneous otoacoustic emissions during and after postural change. J Acoust Soc Am 107:3308-3316

Gea SLR, Decraemer WF, Funnell RWJ, Dirckx JJJ, Maier H (2010) Tympanic membrane boundary deformations derived from static displacements observed with computerized tomography in human and gerbil. J Assoc Res Otolaryngol 11:1-17

Glasberg BR, MOORE BCJ (1990) Derivation of auditory filter shapes from notched-noise data. Hear Res 47:103-138

Hauser R, Probst R, Harris FP (1993) Effects of atmospheric pressure variation on spontaneous, transiently evoked, and distortion product otoacoustic emissions in normal human ears. Hear Res 69:133-145

Hof JR, Anteunis LJC, Chenault MN and Van Dijk P (2005) Otoacoustic emissions at compensated middle ear pressure in children. Int J Audiol

Homma K, Shimizu Y, Kim N, Du Y, Puria S (2010) Effects of ear-canal pressurization on middle-ear bone- and air-conduction responses. Hear Res 263:204-215

Jones K, Tubis A, Long GR, Burns EM, Strickland EA (1986) Interactions among multiple spontaneous otoacoustic emissions. In: Allen JB, Hall JL, Hubbard A, Neely ST, Tubis A (eds) Peripheral auditory mechanisms. Springer, Berlin, pp 266-273

LeE C-Y, Rosowski JJ (2001) Effects of middle-ear static pressure on pars tensa and pars flaccida of gerbil ears. Hear Res 153:146-163

Long GR, Talmadge CL (1997) Spontaneous otoacoustic emission frequency is modulated by heartbeat. J Acoust Soc Am 102:28312848
Murphy WJ, Talmadge CL, Tubis A, Long GR (1995a) Relaxation dynamics of spontaneous otoacoustic emissions perturbed by external tones. I. Response to pulsed single-tone suppressors. J Acoust Soc Am 97:3702-3710

Murphy WJ, Talmadge CL, Tubis A, Long GR (1995b) Relaxation dynamics of spontaneous otoacoustic emissions perturbed by external tones. II. Suppression of interacting emissions. J Acoust Soc Am 97:3711-3720

Schloth E, Zwicker E (1983) Mechanical and acoustical influences on otoacoustic emissions. Hear Res 11:285-293

SHera CA (2003) Mammalian spontaneous otoacoustic emissions are amplitude-stabilized cochlear standing waves. J Acoust Soc Am 114:244-262

Stratonovich RL (1963) Topics in the theory of random noise, volume 2. Gordon and Breach, New York

Talmadge C, Long G, Murphy W, Tubis A (1993) New off-line method for detecting spontaneous otoacoustic emissions in human subjects. Hear Res 71:170-182

VAn Dijk P, Wit HP (1990a) Amplitude and frequency fluctuations of spontaneous otoacoustic emissions. J Acoust Soc Am 88:1779-1793

VAN DijK P, Wit HP (1990b) Synchronization of spontaneous otoacoustic emissions to a $2 f_{1}-f_{2}$ distortion product. J Acoust Soc Am 88:850-856

Van Dijk P, Wit HP, Segenhout JM, Tubis A (1994) Wiener kernel analysis of inner ear function in the American bullfrog. J Acoust Soc Am 95:904-919

Voss Se, Adegoke MF, Horton NJ, Sheth KN, Rosand J, Shera CA (2010) Posture systematically alters ear-canal reflectance and DPOAE properties. Hear Res 263:43-51

Wada H, Ohyama K, Kobayashi T, Koike T, Noguchi S (1995) Effect of middle ear on otoacoustic emissions. Audiology 34:161-176

Zweig G, SHera CA (1995) The origin of periodicity in the spectrum of evoked otoacoustic emissions. J Acoust Soc Am 98:2018-2047 